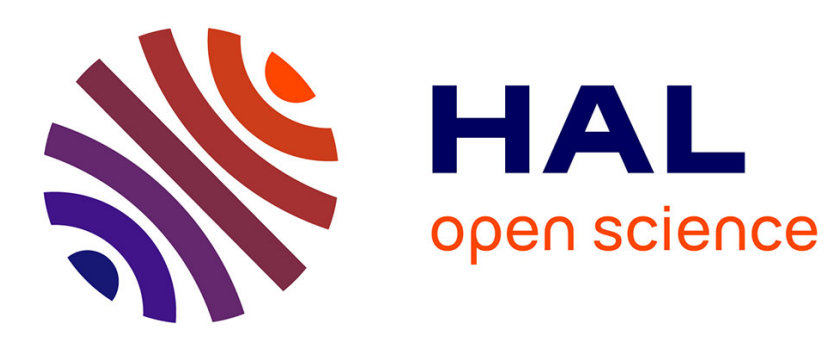

\title{
Fluid flow and effective permeability of an infinite matrix containing disc-shaped cracks
}

\author{
Ahmad Pouya, Minh-Ngoc Vu
}

\section{To cite this version:}

Ahmad Pouya, Minh-Ngoc Vu. Fluid flow and effective permeability of an infinite matrix containing disc-shaped cracks. Advances in Water Resources, 2012, 42, pp.37-46. 10.1016/j.advwatres.2012.03.005 . hal-00700099

\section{HAL Id: hal-00700099 \\ https://hal.science/hal-00700099}

Submitted on 22 May 2012

HAL is a multi-disciplinary open access archive for the deposit and dissemination of scientific research documents, whether they are published or not. The documents may come from teaching and research institutions in France or abroad, or from public or private research centers.
L'archive ouverte pluridisciplinaire HAL, est destinée au dépôt et à la diffusion de documents scientifiques de niveau recherche, publiés ou non, émanant des établissements d'enseignement et de recherche français ou étrangers, des laboratoires publics ou privés. 
Advances in Water Resources 42 (2012) 37-46

http://dx.doi.org/10.1016/j.advwatres.2012.03.005

Fluid flow and effective permeability of an infinite matrix containing disc-shaped cracks

\author{
Ahmad Pouya ${ }^{1}$, Minh-Ngoc $\mathrm{Vu}^{2,3}$ \\ 1.Université Paris-Est, Laboratoire Navier (ENPC/IFSTTAR/CNRS), IFSTTAR, 58 bd. Lefebvre, 75732 Paris, \\ France \\ 2. BRGM/RNSC F-45060 Orléans, Cedex 2 France/ 3. Le Quy Don Technical University, 100 Hoang Quoc \\ Viet, Hanoi, Vietnam \\ ahmad.pouya@enpc.fr
}

\begin{abstract}
A basic equation governing the steady-state flow around a single crack in an infinite porous body is given. The flow through the crack obeys to the Poiseiulle's law and the matrix has an anisotropic permeability. A semi-analytical solution is established for this equation in the case of elliptical disc-shaped crack. This solution takes a closed-form expression for the case of superconducting circular cracks. The results are compared to those obtained for flattened ellipsoidal inclusions obeying to the Darcy's flow law, which are in some works supposed to represent the cracks. It is shown that the flow solution for an elliptical disc-shaped crack obeying to the Poiseuille's law is different from that obtained as the limiting case of flattened ellipsoidal inclusions. The results are then used to establish dilute Mori-Tanaka and selfconsistent estimates of the effective permeability of porous media containing Poiseuille's type elliptical cracks.
\end{abstract}

Keywords: porous media, crack, steady-state flow, Poiseuille's law, Darcy's law, effective permeability, upscaling

\title{
1 Introduction
}

The effective permeability of fractured rocks and micro-cracked porous materials is a great interest for many environmental and industrial applications such as hydrology, petroleum engineering, nuclear waste disposal, geothermal expansion.... Numerous studies dealt with permeability of fracture network in three-dimensional media. Koudina et al. [[1] presented a method to generate a triangular mesh on three-dimensional network of polygonal fracture surfaces, and used the finite volume method to compute the permeability of fractured media. The numerical tool developed in this way was employed to study the effective permeability of fractures network with statistical distribution [[2]. However, the rock matrix was supposed to be impervious in these works which does not correspond to some real cases. Therefore, other theoretical and numerical investigations have focused on this problem $[[3,[4[5[6,[7$, [8]. A fundamental problem for modelling the effective permeability of cracked materials is 
the determination of the steady state flow around a single crack in an infinite homogeneous matrix. In this work, we are interested in the case of cracks and fractures that can be represented geometrically by zero thickness surfaces with non pressure jump between their two faces, which corresponds to an assumption of zero cross section resistance for the fracture. Therefore, we do not consider the cases of non-equilibrium flow [[9] or of impervious fracture [[10]. In many physical studies, the Poiseuille's law is chosen preferentially to represent the flow in the cracks. The paper devotes to determine flow solution around a single crack obeying to the Poiseuille's law that is the key issue for determining the effective permeability.

The flow in cracks, embedded in an infinite matrix, has been determined in some works by using a model of ellipsoidal inclusion, in which the flow is governed by Darcy's law [[5[11]. It is implicitly supposed in these works that the flow in the crack with the Poiseuille's law is obtained as the limiting case of a flattened ellipsoidal inclusion with thickness tending to zero. In this study, we show that this is not true, the solution obtained for the flow in and around an elliptical disc-shaped crack obeying to the Poiseuille's law is different from that obtained as a limit of ellipsoidal inclusions obeying to Darcy's law. Only some partial results, for instance, the pressure field in the case of superconducting cracks, are similar in the two problems.

To determine the solution of flow in a matrix containing a single elliptical disc-shaped crack, we use recent theoretical results established for the more general problem of multiple intersecting fracture surfaces [[12]. The potential solution obtained for this general problem, as an extension of preceding works for the 2D case [[13[14], is based on singular integral equations allowing to reduce the dimension of the problem from $3 \mathrm{D}$ to $2 \mathrm{D}$ and, hence, particularly interesting for numerical methods. However, it will be seen in the sequel that for the case of a single elliptical disc-shaped crack, it allows also deriving semi-analytical solutions as an alternative way to the use of harmonic functions $[15,16]$ or of dual integral equations methods $[17,18]$.

The solution obtained for the single crack will be then used to estimate the effective permeability of the cracked porous materials by some classical upscaling methods.

\section{Notations :}

In the sequel, light-face (Greek or Latin) letters denote scalars; underlined letters denote vectors, bold-face letters designate second rank tensors or double-index matrices. The scalar product of two vectors $\underline{a}$ and $\underline{b}$ is labelled as $\underline{a} . \underline{b}$. For second rank tensors, the tensor transposed from $\boldsymbol{A}$ is denoted $\boldsymbol{A}^{\mathrm{T}}$, the matrix product is labelled as $\boldsymbol{A} \boldsymbol{B}$ and the determinant as $|\boldsymbol{A}|$. The operation of $\boldsymbol{A}$ on $\underline{a}$ is labelled as $\boldsymbol{A} . \underline{a}$. The convention of summation on repeated indices is not used for Latin indices $(i, j, k \ldots)$ that number surfaces, lines, etc., and are noted indifferently as subscript or upperscript. $\nabla$ represents the gradient and $\Delta$ the Laplace operator for a scalar field and $(\nabla$.) the divergence for a vector field.

\section{Governing equations}

An infinite porous 3D body $\Omega$ containing an elliptical disc-fracture surface $\Gamma$ is considered (Figure 1). The points of $\Omega$ are denoted by $\underline{x}=\left(x_{1}, x_{2}, x_{3}\right)$ and, when they are located on the crack surface, by $\underline{z}=\left(z_{1}, z_{2}, z_{3}\right)$. The surface $\Gamma$ is defined by a function $\underline{z}(\underline{s})$ from $\mathbb{R}^{2} \rightarrow \mathbb{R}^{3}$ parameterized by $\underline{s}=\left(s_{1}, s_{2}\right)$. Fluid flow through the matrix is governed by Darcy's law: 
where, $\boldsymbol{k}$ is the permeability tensor of porous matrix supposed to be uniform; $\underline{v}(\underline{x})$ and $p(\underline{x})$ the local velocity and pressure, respectively.

The local mass balance in the matrix reads:

$\forall \underline{x} \in \Omega-\Gamma$

$$
\nabla \cdot \underline{v}(\underline{x})=0
$$

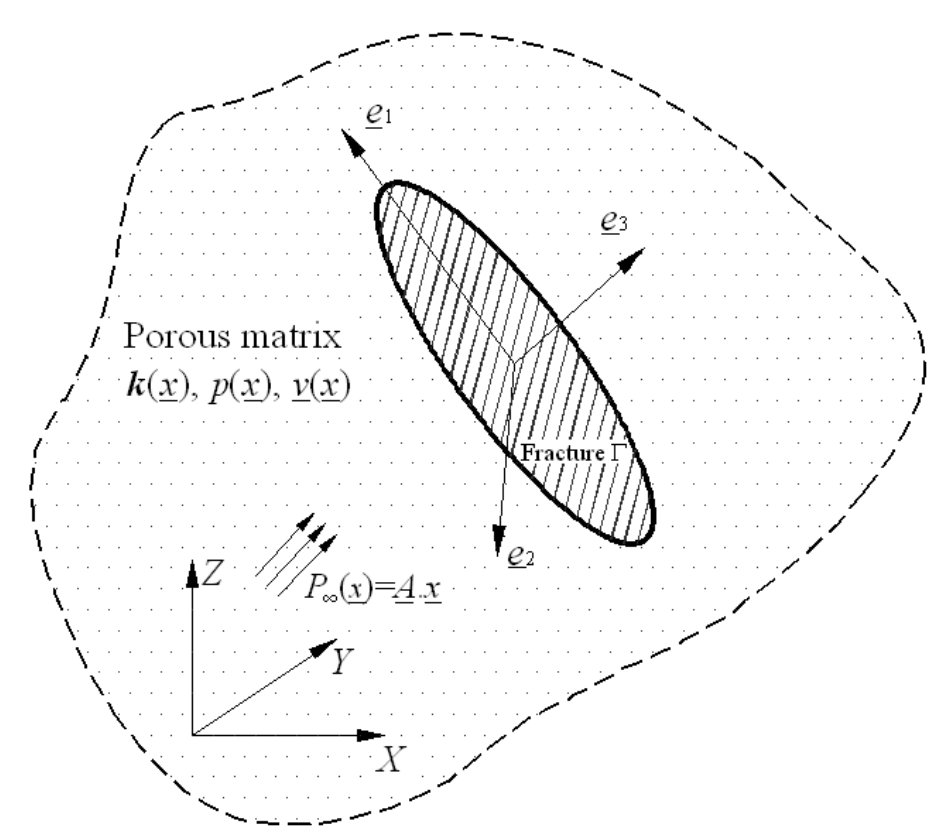

Figure 1: An infinite porous medium containing a flat elliptical disc shaped crack

The flow in the fracture surface is characterized by a pressure field $p(\underline{s})$ and an infiltration field $q(\underline{s})$ in this surface. The total infiltration $q(\underline{s})$ through a fracture section is determined by the integral of fluid velocity $\underline{v}$ over this physical section of the thickness $2 e$ as follows:

$$
\forall \underline{s} \in \Gamma \quad \underline{q}(\underline{s})=\int_{-e}^{e} \underline{v}(\underline{s}, y) d y
$$

The crack-matrix mass exchange at a point on the fracture surface excluded its boundary is established by considering the mass balance in a small volume surrounding this point and reads [[12]:

$\forall \underline{x} \in \Gamma-L ;$

$$
\llbracket \underline{v}(\underline{x}) \rrbracket \cdot \underline{n}(\underline{s})+\nabla_{s} \cdot \underline{q}(\underline{s})=0
$$

where, $\nabla_{s} \underline{q} \underline{q}(\underline{s})$ is the surface divergence and $L$ the set of points on the crack boundaries. In addition, analysis of mass balance around a point on the crack boundary leads to the following mass balance condition at this point:

$\forall \underline{z}(\underline{s}) \in L ;$

$$
\underline{q}(\underline{s}) \cdot \underline{m}(\underline{s})=0
$$


In this equation, $q$ is the infiltration vector in the crack and $\underline{m}$ is the outward unit normal vector of the boundary line that is tangent to the crack plane.

The body is submitted at its infinite boundary to a pressure field $p_{\infty}(\underline{x})$ :

$$
\lim _{\|x\| \rightarrow \infty}\left[p(\underline{x})-p_{\infty}(\underline{x})\right]=0
$$

$p_{\infty}$ satisfies $\Delta p_{\infty}=0$. For permeability upscaling, a constant pressure gradient is supposed to be prescribed to the matrix at its infinite boundary. Designating by $\underline{A}$ the far-field pressure gradient, we have:

$$
p_{\infty}(\underline{x})=\underline{A} \cdot \underline{x}
$$

The equations to be satisfied by $p$ are the boundary condition (6), mass balances (2), (4), (5) and the constitutive equation (1). The solution obtained by Pouya [[12] expresses the pressure field in the whole matrix as a function of the infiltration vector in the fracture as follows:

$$
p(\underline{x})=p_{\infty}(\underline{x})+\frac{1}{4 \pi \sqrt{|\boldsymbol{k}|}} \int_{\Gamma} \frac{\underline{q}(\underline{s}) \cdot \boldsymbol{k}^{-1} \cdot(\underline{x}-\underline{z}(\underline{s}))}{\left[(\underline{x}-\underline{z}(\underline{s})) \cdot \boldsymbol{k}^{-1} \cdot(\underline{x}-\underline{z}(\underline{s}))\right]^{3 / 2}} d s
$$

In this equation, $d s$ represents the surface element on $\Gamma$. This solution satisfies well the farfield condition (6). Also, noting that the integrant function in the right-hand side integral is equal to $-\underline{q}(\underline{s}) \cdot \nabla_{\underline{x}}(1 / r)$ with $r=\left[(\underline{x}-\underline{z}) \cdot k^{-1} \cdot(\underline{x}-\underline{z})\right]^{1 / 2}$, the mass balance (2) can be shown for $\underline{x}$ non situated on the fracture. To establish that (4) is well satisfied by (8) is little more technical and we refer to [[12] for its demonstration.

It should be noted that the only significant assumptions limiting the validity of this solution are that matrix permeability $\boldsymbol{k}$ is assumed to be uniform and that there is no pressure gap between the two sides of the crack surface. Although we consider in the sequel only the case of a plane crack surfaces, the solution (8) remains valid for curved-surface fractures, and, in its general form given in [[12], for multiple intersecting fractures.

Also, in equation (8), the constitutive flow law in the fracture is not constrained. A linear relation between the infiltration vector and the pressure gradient, as an extension of the Poiseuille's law for the viscous fluid flow between two parallel plates, is widely used in the literature to represent the flow in the crack:

$$
\underline{q}(\underline{s})=-c(\underline{s}) \cdot \nabla_{s} p(\underline{s})
$$

The conductivity tensor $\boldsymbol{c}$ depends on the fluid dynamic viscosity $\mu$, the hydraulic aperture of the fracture $e$, and also the roughness of fracture surfaces. In most cases, $c$ is supposed isotropic (in the fracture surface) and replaced by a scalar $c$. In particular, the Poiseuille's law, widely used for flow through rock fractures [[19], determines $c$ from a laminar flow between two parallel plates separated by a constant distance $e$ and leads to $c=e^{3} /(12 \mu)$. However, $\boldsymbol{c}$ can be anisotropic in some cases as shown experimentally by Gentier et al.[[20]. Introducing (9) into (8) leads to an integral equation involving only the unknown pressure field. A simple analysis shows that the solution for a constant pressure gradient along the disc surface, corresponding to the Eshelby-type ellipsoidal inclusion with Dracy's law, does not satisfy this equation. The comparison between the Posieulle-type crack and the flattened ellipsoidal inclusion with Darcy's law will be discussed further in this paper. 
However, in this paper we are interested in the case of superconducting cracks corresponding to the limiting case $c \rightarrow \infty$. This is an important limiting case, currently addressed in the literature because it allows determining the upper bound of the effective permeability for fixed crack density and geometry.

\section{Superconductive elliptical crack-inclusion}

In the case of superconducting fracture, the pressure remains constant along the fracture surface. We consider an elliptical disc shaped crack $D$ and denote by $d_{1}$ and $d_{2}$ the halfdiameters of the ellipse, by the unit vectors $\underline{e}_{1}$ and $\underline{e}_{2}$ its principal directions and by $\underline{e}_{3}$ the unit normal to the crack plane. A coordinate system is defined with its origin located at the crack centre and its axes parallel to $\underline{e}_{1}, \underline{e}_{2}$ and $\underline{e}_{3}$. The equation of the ellipse reads $\underline{x} \cdot \boldsymbol{B} \cdot \underline{x}=1$ for $\underline{x} \cdot \underline{e}_{3}$ $=0$. We note:

$$
\lambda_{1}=1 / d_{1}^{2}, \quad \lambda_{2}=1 / d_{2}^{2}, \quad \boldsymbol{B}=\lambda_{1} \underline{e}_{1} \otimes \underline{e}_{1}+\lambda_{2} \underline{e}_{2} \otimes \underline{e}_{2}
$$

The general result needed for permeability upscalling is the integral of the infiltration over the disc surface. This integral is a linear function of the far-field gradient $\underline{A}$ and can be written as $\boldsymbol{\Psi} . \underline{A}$, where the tensor $\boldsymbol{\Psi}$ is a function of $\boldsymbol{c}, \boldsymbol{k}$ and $\boldsymbol{B}$. By application of the linear transformation [[12], the problem can be changed into that of an ellipsoidal disc in an isotropic matrix. Nevertheless, a numerical method is required to solve the general problem for the case of finite and anisotropic conductivity tensor $\boldsymbol{c}$. In the following, we consider the particular case of superconducting crack, $c \rightarrow \infty$, that allows us to derive an analytical or semi-analytical solution.

The equation (8) written for a single elliptical crack $D$ embedded in an infinite porous matrix with isotropic permeability $k$ yields:

$$
p(\underline{x})=\underline{A} \cdot \underline{x}-\frac{1}{4 \pi k} \int_{D} \frac{\underline{q}(\underline{\xi}) \cdot(\underline{\xi}-\underline{x})}{\|\underline{\xi}-\underline{x}\|^{3}} d \xi
$$

In this equation, $d \xi$ represents the surface element on $D$. The general solution can be obtained by superposition method. It requires three elementary solutions for $\underline{A}$ parallel to $\underline{e}_{1}, \underline{e}_{2}$ and $\underline{e}_{3}$. A simple analysis shows that for $\underline{A}$ parallel to $\underline{e}_{3}$ the solution is a uniform gradient pressure not perturbed by the presence of the crack, i.e., $p(\underline{x})=\underline{A} . \underline{x}$ in the whole body. To build the complete solution, it is sufficient to determine the solution for $\underline{A}$ parallel to the $x$-axis. Hence, we try to solve the problem for $p_{\infty}(\underline{x})=A_{1} x_{1}$.

The condition of superconducting crack imposes that the pressure $p$ must be constant in the crack surface, and because of symmetry considerations, this constant is zero. Therefore, designating, for a field $q(\xi)$, by:

$$
I(\underline{q})=\int_{D} \frac{\underline{q}(\underline{\xi}) \cdot(\underline{\xi}-\underline{x})}{\|\underline{\xi}-\underline{x}\|^{3}} d \xi
$$

the solution $q$ must satisfy:

$$
I(q)=4 \pi k A_{1} x_{1}
$$




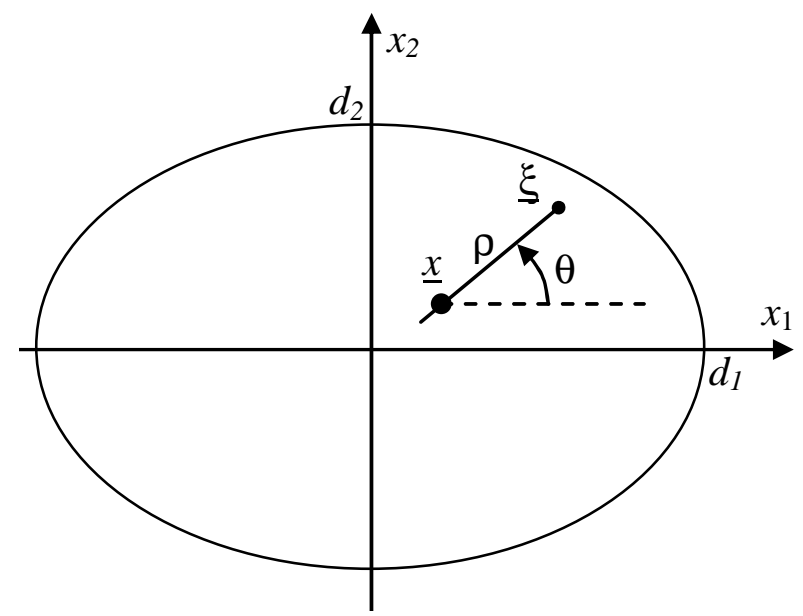

Figure 2: Geometrical parameters defining the collocation point $\underline{x}$ and the integration point $\xi$ in the elliptical crack surface

To find a solution for this problem, we try first the following expression which is inspired by the 2D solution given by Pouya \& Ghabezloo [[14]:

$$
\underline{q}^{0}(\underline{\xi})=h_{1} \sqrt{1-\underline{\xi} \cdot \boldsymbol{B} \cdot \underline{\xi}} \underline{e}_{1}
$$

As shown in Appendix A, the expression found for $I$ is a linear function of $x_{1}$ for $q=q^{0}$ which allows us to build the solution for the equation (13). The general expression found for this solution has recourse to the complete elliptic integrals of first and second kind $K(\lambda)$ and $E(\lambda)$ defined by:

$$
K(\lambda)=\int_{\theta=0}^{\pi / 2} \frac{d \theta}{\sqrt{1-\lambda^{2} \sin ^{2} \theta}}, \quad E(\lambda)=\int_{\theta=0}^{\pi / 2} \sqrt{1-\lambda^{2} \sin ^{2} \theta} d \theta \quad(0 \leq \lambda \leq 1)
$$

The semi-analytical or iterative numerical methods used to calculate these complete elliptic integrals $K(\lambda)$ and $E(\lambda)$ can be found in mathematical handbooks [[21].

Moreover, we define:

$$
\left\{\begin{array}{l}
\phi_{1}\left(\lambda_{1}, \lambda_{2}\right)=\int_{\theta=0}^{\pi / 2} \frac{\lambda_{1} \cos ^{2} \theta}{\sqrt{\lambda_{1} \cos ^{2} \theta+\lambda_{2} \sin ^{2} \theta}} d \theta \\
\phi_{2}\left(\lambda_{1}, \lambda_{2}\right)=\int_{\theta=0}^{\pi / 2} \frac{\lambda_{2} \sin ^{2} \theta}{\sqrt{\lambda_{1} \cos ^{2} \theta+\lambda_{2} \sin ^{2} \theta}} d \theta
\end{array}\right.
$$

It can be noticed that, if $d_{1} \geq d_{2}$, denoting by $\lambda=\sqrt{1-\left(\frac{d_{2}}{d_{1}}\right)^{2}}$, we have: 


$$
\left\{\begin{array}{l}
\phi_{1}\left(\lambda_{1}, \lambda_{2}\right)+\phi_{2}\left(\lambda_{1}, \lambda_{2}\right)=\frac{1}{d_{2}} E(\lambda) \\
d_{1}^{2} \phi_{1}\left(\lambda_{1}, \lambda_{2}\right)+d_{2}^{2} \phi_{2}\left(\lambda_{1}, \lambda_{2}\right)=d_{2} K(\lambda)
\end{array}\right.
$$

This system of equations allows the determination of $\phi_{1}$ and $\phi_{2}$ as function of $K(\lambda)$ and $E(\lambda)$. Then, for $q=q^{0}$, it is shown in Appendix A that:

$$
I=-2 h_{1} \pi \phi_{1}\left(\lambda_{1}, \lambda_{2}\right) x_{1}
$$

As a result, (13) is verified if we take $h_{1}=\frac{-2 k}{\phi_{1}} A_{1}$, and therefore:

$$
\underline{q}^{0}(\underline{\xi})=-\frac{2 k}{\phi_{1}} A_{1} \sqrt{1-\underline{\xi} \cdot \boldsymbol{B} \cdot \underline{\xi}} \underline{e}_{1}
$$

According to this expression, the flux $q^{0}$ in the crack has a constant direction parallel to the far-field gradient, as in the case of ellipsoidal Darcy type inclusions. However, $q^{0}$ is not the real solution of the flow in the disc. As a matter of fact, it must be noted that the solution of the equation (13) is not unique. If the vector $\underline{\omega}(\xi)$ satisfies $\underline{\nabla} \cdot \underline{\omega}=0$ in the disc and $\underline{\omega} . \underline{n}=0$ on its boundary where $\underline{n}$ is outward unit normal vector to the boundary, then we calculate:

$$
I(\underline{\omega})=-\int_{\Gamma} \underline{\omega}(\underline{\xi}) \cdot \underline{\nabla}\left(\|\underline{\xi}-\underline{x}\|^{-1}\right) d \xi=-\int_{\partial \Gamma}\|\underline{\xi}-\underline{x}\|^{-1} \underline{\omega}(\underline{\xi}) \cdot \underline{n} d s+\int_{\Gamma}\|\underline{\xi}-\underline{x}\|^{-1} \underline{\nabla} \cdot \underline{\omega} d \underline{\xi}=0
$$

Consequently, $q=q^{0}+\underline{\omega}$ also satisfies (13). This degeneracy of the solution can be removed if we suppose that the superconducting crack is the limit case of a crack with the conductivity $\boldsymbol{c}=\sigma \boldsymbol{c}_{0}$ where $\boldsymbol{c}_{0}$ is constant and $\sigma \rightarrow \infty$. This implies that $\boldsymbol{c}_{0}^{-1} q$ must be a gradient field (pressure gradient) and satisfy the condition of symmetry of crossed derivatives. This condition allows us to determine a unique $q$ saved that $\boldsymbol{c}_{0}$ is symmetric and positive definite. In the case of a superconducting crack considered as the limit case of an isotropic conductivity crack, this condition implies that $q$ has to be a gradient field. It can be checked that $q^{0}$ given by (19) is not a gradient field. A complementary part $\underline{\omega}(\xi)$ should be added to $q^{0}$ to make it a gradient field satisfying (13). Thus, the solution of the infiltration field $q$ reads:

$$
q(\xi)=q^{0}(\xi)+\underline{\omega}(\xi)
$$

where, the complementary part $\underline{\omega}$ has to satisfy the following conditions:

$$
\begin{array}{ll}
\forall \xi \in D ; & \underline{\nabla} \cdot \underline{\omega}=0 \\
\forall \xi \in \partial D ; & \underline{\omega} \cdot \underline{n}=0 \\
\forall \xi \in D ; & \partial_{1}\left(q^{0}{ }_{2}+\omega_{2}\right)=\partial_{2}\left(q^{0}{ }_{1}+\omega_{1}\right)
\end{array}
$$

The last condition assures that $q$ is a gradient field. Mathematically, equation (22) is satisfied if we take:

$$
\omega_{1}=\partial_{2} \varphi, \quad \omega_{2}=-\partial_{1} \varphi
$$

with $\varphi$ an arbitrary function. Then, the condition (24) yields: 


$$
\Delta_{s} \varphi=\frac{2 k A_{1}}{\phi_{1}} \partial_{2} \sqrt{1-\underline{\xi} \cdot \boldsymbol{B} \cdot \underline{\xi}}
$$

where, $\Delta_{s} \varphi=\left(\partial_{1}^{2}+\partial_{2}^{2}\right) \varphi$. Substituting (25) into (23) and rearranging the terms, we find:

$\forall \xi \in \partial D ; \quad \nabla \varphi \cdot \underline{t}=0$

where $\underline{t}$ is the unit tangent vector to the crack boundary $\partial D$. This equation implies that $\varphi$ is constant on the boundary, hence:

$\forall \xi \in \partial D ; \quad \varphi=\varphi_{0}$

with, $\varphi_{0}$ a constant. A solution for the Poisson's equation (26) over the elliptical domain exists for every Dirichlet boundary condition and in particular (28) with constant $\varphi_{0}$. We can take $\varphi_{0}$ $=0$ because the addition of a constant value to $\varphi$ does not change the solution $\omega$. The solution of equation (26) with $\varphi=0$ on the boundary can be determined numerically in the general case and analytically in some special cases. Two particular cases in which the solution can be derived analytically are the following:

\section{Case 1: Limit of infinitely long elliptical disc}

We suppose the limit case of elliptical disc with infinite extension in the direction 2 . Geometrically, this corresponds to an infinite band of width $2 d_{1}$ parallel to the direction 2 . The solution, for a far-field gradient $A_{1}$ parallel to $\underline{e}_{1}$ corresponds to that of the $2 \mathrm{D}$ case with a crack of length 2D in the plane $\left(x_{1}, x_{2}\right)$ parallel to $x_{1}$. The solution can therefore be compared to that given for this case by Pouya \& Ghabezloo [[14]. In the 3D problem, $d_{2} \rightarrow \infty$ and $\lambda_{2}=0$ and $\underline{q}^{0}(\underline{\xi})=-2 k \sqrt{d_{1}^{2}-\xi_{1}^{2}} A_{1} \underline{e}_{1}$ is a gradient field. Thus, $\underline{\omega}=0$. The exact solution is then $\underline{q}(\underline{\xi})=-2 k \sqrt{d_{1}^{2}-\xi_{1}^{2}} A_{1} \underline{e}_{1}$ which is exactly the solution found in $2 \mathrm{D}$ case by Pouya \& Ghabezloo [[14].

\section{Case 2: Circular crack with radius $R$}

In this case, $d_{1}=d_{2}=R$. We find $\phi_{1}=\phi_{2}=\frac{\pi}{4 R}$ and $\underline{q}^{0}(\underline{\xi})=\frac{8 k A_{1}}{\pi} \sqrt{R^{2}-r^{2}} \underline{e}_{1}$ with $r^{2}=\xi . \xi$. In order to find the expression of the complementary function $\underline{\omega}$, we look for a solution of (26) in the following form:

$$
\varphi(\xi)=\chi(\xi)+\partial_{2} \zeta(r)
$$

where, $\zeta(r)$ satisfies:

$$
\Delta_{s} \zeta=-\frac{8 k A_{1}}{\pi} \sqrt{R^{2}-r^{2}}
$$

Then, $\chi$ has to satisfy $\Delta \chi=0$. The general solution of (30) is:

$$
\zeta(r)=-\frac{8 k A_{1}}{\pi}\left[-\frac{1}{3} R^{2} \sqrt{R^{2}-r^{2}}-\frac{1}{6} R^{3} \operatorname{Ln} \frac{R-\sqrt{R^{2}-r^{2}}}{R+\sqrt{R^{2}-r^{2}}}-\frac{1}{9}\left(R^{2}-r^{2}\right)^{3 / 2}+C_{1} \operatorname{Ln} r+C_{2}\right]
$$


where, $C_{1}, C_{2}$ are two constants. Thus,

$$
\partial_{2} \zeta=-\frac{8 k A_{1}}{\pi} \frac{\xi_{2}}{r^{2}}\left[\frac{1}{3}\left(R^{2}-r^{2}\right)^{3 / 2}-C_{1}\right]
$$

Substituting this expression in (29) and taking into account the boundary condition $\varphi(R)=0$, we find that $\chi=-C_{1} \frac{8 k A_{1}}{\pi} \frac{\xi_{2}}{R^{2}}$ on the boundary. Hence, the solution of the equation $\Delta \chi=0$ is $\chi(\underline{\xi})=-C_{1} \frac{8 k A_{1}}{\pi} \frac{\xi_{2}}{R^{2}}$ and therefore the solution for $\varphi$ is:

$$
\varphi=-\frac{8 k A_{1}}{\pi} \frac{\xi_{2}}{r^{2}}\left[\frac{1}{3}\left(R^{2}-r^{2}\right)^{3 / 2}-C_{1}\right]-C_{1} \frac{8 k A_{1}}{\pi} \frac{\xi_{2}}{R^{2}}
$$

The constant $C_{1}$ is determined by the condition that $q$ has to be finite in the crack or that $\varphi$ is not singular when $r \rightarrow 0$. This imposes $C_{1}=\frac{R^{3}}{3}$. Substituting by this expression in (25) and (21) we find the solution of infiltration in the circular disc under the far-field pressure gradient $A_{1}$ as follows:

$$
\begin{aligned}
\underline{q}(\underline{\xi})= & -\frac{8 k A_{1}}{\pi}\left[\frac{\xi_{1}^{2}}{r^{2}} \sqrt{R^{2}-r^{2}}-\frac{\xi_{1}^{2}-\xi_{2}^{2}}{3 r^{4}}\left(R^{3}-\left(\sqrt{R^{2}-r^{2}}\right)^{3}\right)+\frac{R}{3}\right] \underline{e}_{1} \\
& -\frac{8 k A_{1}}{\pi}\left[-\frac{2 \xi_{1} \xi_{2}}{3 r^{4}}\left(R^{3}-\left(\sqrt{R^{2}-r^{2}}\right)^{3}\right)+\frac{\xi_{1} \xi_{2}}{r^{2}}\left(R^{2}-r^{2}\right)^{1 / 2}\right] \underline{e}_{2}
\end{aligned}
$$

The two components $q_{1}(\xi)$, parallel to the far-field gradient and $q_{2}(\xi)$, normal to this gradient, are represented in the Figure 3 for $A_{1}=1$ and $k=1$.
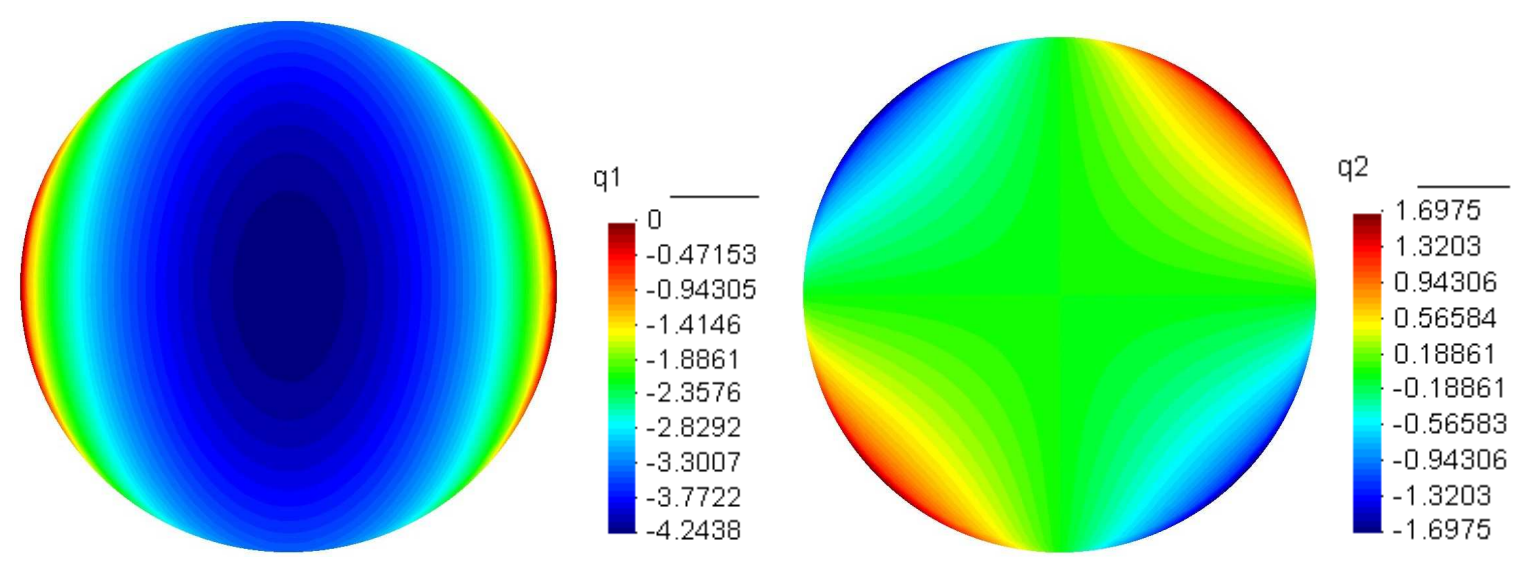

Figure 3: Closed-form solution of infiltration $q$ in fracture of unit radius in an infinite isotropic matrix under a far-field pressure gradient in $x_{1}$-direction: $q_{1}$ component parallel to the far-field gradient, $q_{2}$ orthogonal to the far-field gradient 
The elementary solution for a far-field pressure gradient $A_{2}$ parallel to $\underline{e}_{2}$ is obtained from (34) by index changing $1 \leftrightarrow 2$. As mentioned above, the solution for the general case of a far-field pressure gradient $\underline{A}$ is obtained by superposition of the three elementary solutions. By introducing the notation:

$$
\begin{aligned}
& r=\sqrt{\underline{\xi} \cdot \underline{\xi}}, \quad \underline{\hat{\xi}}=\frac{1}{r} \underline{\xi} \\
& \boldsymbol{T}=\boldsymbol{\delta}-\underline{n} \otimes \underline{n}
\end{aligned}
$$

where $\underline{n}$ is the unit normal vector to the crack plane, the general solution obtained for $q$ has the following expression:

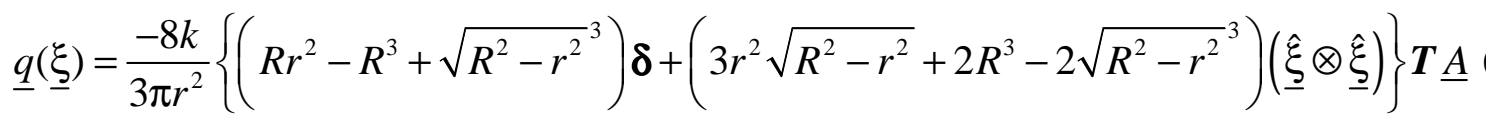

It is important to note here a fundamental difference between this result and what could be obtained as the limiting case of flow solution in a flattened ellipsoidal inclusion. The comparison between the two models is presented farther in the paper. But it is already possible to note the flow in the elliptical crack given by (37), as seen also in the Fig. 3, is not uniform and parallel to the farfield gradient contrary to the case of ellipsoidal inclusions in which the fluid velocity is found to be uniform and parallel, at least if the matrix and inclusion are isotropic, to the farfield gradient. More precisely, for instance for a far-filed pressure gradient parallel to the $x$-axis, the solution given by (37) has a non vanishing component in the $y$-axis direction as shown by the expression (34) (see the figure 3 in the paper). However, for the same problem modelled by a flattened spheroidal inclusion, the fluid velocity in the inclusion would be parallel to the $x$-axis, so with non component in the $y$-axis direction. This shows the fundamental difference between the two types of flow and the fact that the solution for the Poiseuille crack can not be obtained as the limiting case of ellipsoidal inclusions with Darcy's law.

The solution (37) could be probably obtained by other mathematical methods for instance based on the use of harmonics functions instead of the potential solution (11). But for the difference underlined here-above, it can not be obtained as a limiting case of solutions presented in the literature for ellipsoidal inclusions obeying Darcy's law.

The solution (37) represents an original and fundamental result that opens the way for further theoretical investigations concerning flow in cracked porous materials.

\section{Pressure field in the matrix}

The solution of the infiltration $q$ through the crack in (37) allows the determination of the pressure field in the matrix by using equation (11).

For the discussions carried on farther in this paper, it is useful to decompose $q$ in $q^{0}$ and $\underline{\omega}$ parts according to (21) and to write:

$$
p(\underline{x})=\underline{A} \cdot \underline{x}-\frac{1}{4 \pi k} \int_{D} \frac{\left[\underline{q}^{0}(\underline{\xi})+\underline{\omega}(\underline{\xi})\right] \cdot(\underline{\xi}-\underline{x})}{\|\underline{\xi}-\underline{x}\|^{3}} d \xi
$$


The equation (20) shows that the contribution of $\underline{\omega}$ to the integral vanishes. Besides, we can deduce from (19), by superposition and by noting that a far-field gradient parallel to $\underline{e}_{3}$ does not induce flow in the fracture, that, for a general far-field gradient $\underline{A}, q^{0}$ is given by:

$$
\underline{q}^{0}(\underline{\xi})=-2 k \sqrt{1-\underline{\xi} \cdot \boldsymbol{B} \cdot \underline{\xi}} \boldsymbol{\Phi} \cdot \underline{A}
$$

where,

$$
\boldsymbol{\Phi}=\left(\phi_{1}\right)^{-1} \underline{e}_{1} \otimes \underline{e}_{1}+\left(\phi_{2}\right)^{-1} \underline{e}_{2} \otimes \underline{e}_{2}
$$

Substituting (40) into (38) yields:

$$
p(\underline{x})=\left(\underline{x}-\frac{1}{2 \pi} \boldsymbol{\Phi} \cdot \int_{D} \frac{\sqrt{1-\underline{\xi} \cdot \underline{B} \cdot \xi}}{\|\underline{\xi}-\underline{x}\|^{3}}(\underline{\xi}-\underline{x}) d \xi\right) \cdot \underline{A}
$$

The pressure field solution given in this way is an explicit integral function of the geometrical parameters of the elliptical disc contained in the tensor $\boldsymbol{B}$. It is interesting to notice that this solution is independent of the matrix permeability $k$. This is due in particular to the assumption of superconducting crack: the pressure solution would depend on $k$ for crack with finite conductivity. Anyway, the equation (41) allows us to determine at least numerically the pressure field around an elliptical crack by direct integration.

For the case of circular disc $\left(\boldsymbol{B}=R^{-2} \boldsymbol{T}\right)$ a closed-form expression for the integral (41) can be derived for a point located on the plane of the crack, i.e. $x_{3}=0$. We find (see Appendix B):

$$
\int_{D} \frac{\sqrt{1-\underline{\xi} \cdot \boldsymbol{B} \cdot \underline{\xi}}}{\|\underline{\xi}-\underline{x}\|^{3}}(\underline{\xi}-\underline{x}) d \xi=\frac{\pi}{2 R}(\psi-\sin \psi) \underline{x}
$$

where, $\psi$ is, as shown in Figure 4, the angular diameter of the disc seen from the position $\underline{x}$.

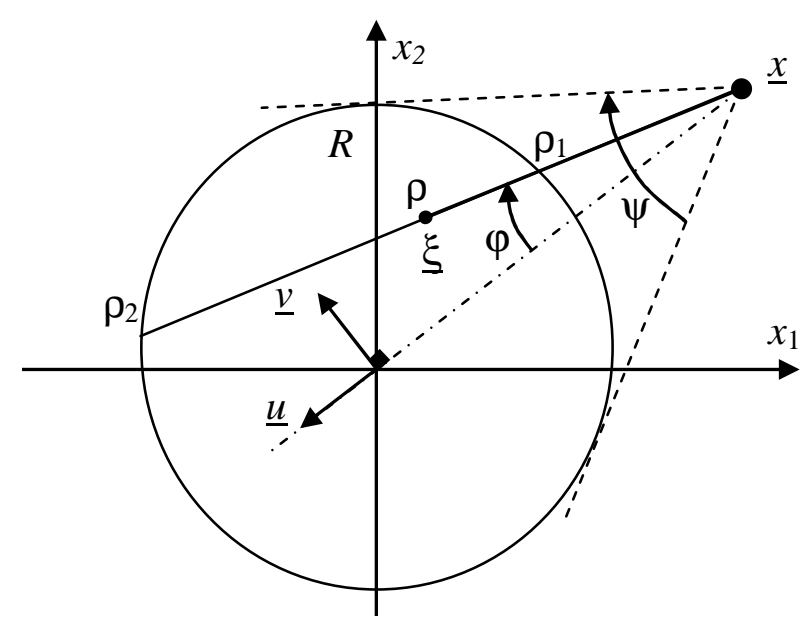

Figure 4: Parameters in polar coordinate system 
Due to superconducting crack, $p(\underline{x})=0$ when $\underline{x}$ is in the crack. We also have $\boldsymbol{\Phi}=(4 R / \pi) \boldsymbol{T}$ and the expression of $p$ for the points exterior to the disc is given by the following expression:

$\underline{x} \cdot \underline{e}_{3}=0 ; \quad p(\underline{x})=\left[1-\frac{1}{\pi}(\psi-\sin \psi)\right] \underline{A} \cdot \underline{x}$

It can be checked that when $\underline{x} \rightarrow \infty$ then $\psi \rightarrow 0$ and so $p(\underline{x}) \rightarrow \underline{A} . \underline{x}$ according to (43). The infinite boundary condition is recovered in this way. Also, when $\underline{x}$ is on the boundary of the disc, $\psi=\pi / 2$ and we find the pressure condition in the disc, $p(\underline{x})=0$. Figure 5 displays the variation of pressure field in the plan containing the superconducting fracture with a far-field pressure gradient $\underline{A}=(1,0,0)$.

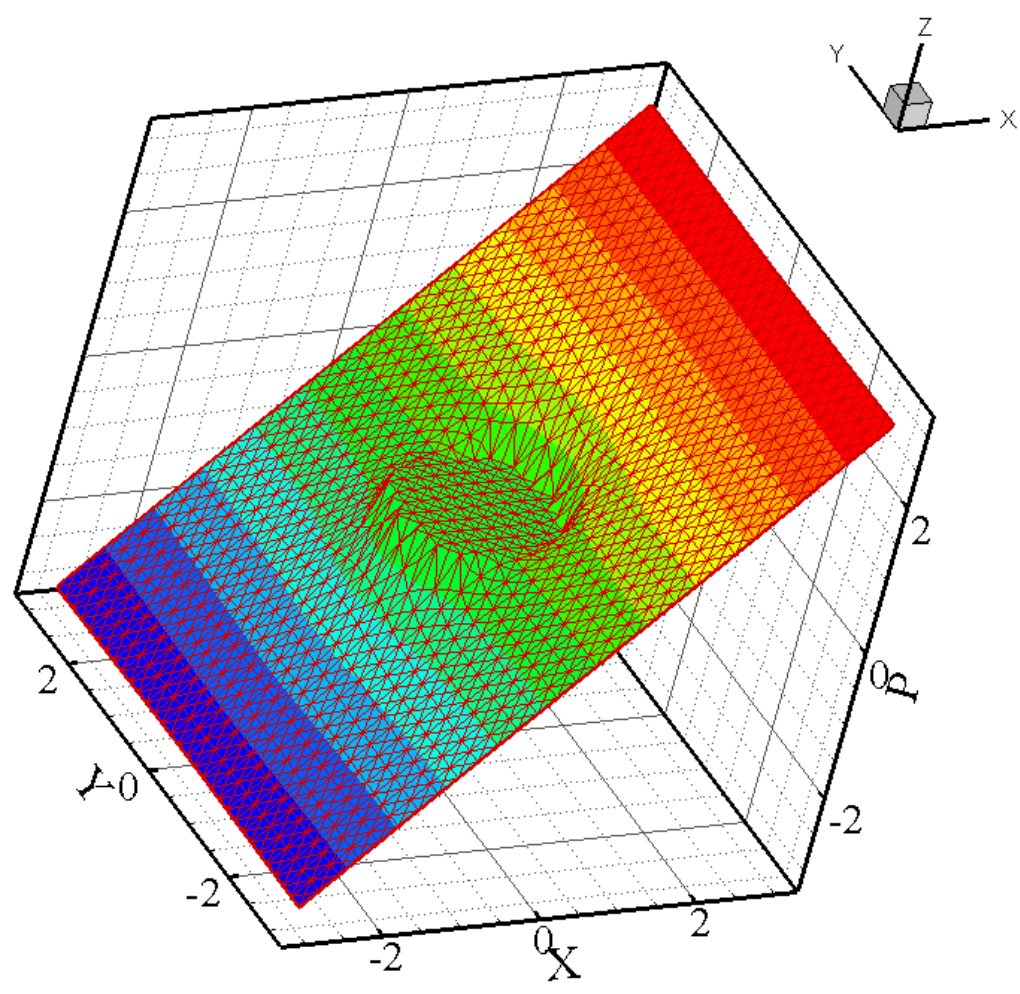

Figure 5: Pressure field around a single superconducting circular disc crack in an infinite matrix prescribed by a constant far-field pressure gradient parallel to $x_{1}$ direction.

It could be noted that this pressure solution could be, in principle, obtained as the limiting case of the pressure field in an infinite matrix containing a superconducting ellipsoidal inclusion. The two problems obey, as a matter of fact, to the same equations and boundary conditions: constant pressure in the crack or inclusion, constant farfield pressure gradient and mass conservation equation in the matrix. The solution could be then obtained as a classical application of potential theories using harmonics functions. However, we have not found in the literature any explicit expression of this solution as given by (43). 


\section{Comparison between Darcy-ellipsoidal inclusions and Poiseulle-crack}

The crack inclusion has been sometimes considered as the limiting case of a penny-shaped inclusion with aspect ratio (ratio between its thickness and diameter) tending to zero. However, a further investigation shows this similarity holds only for some partial results and in restricted cases and this difference seems to be ignored in the literature.

To show this difference, let a circular penny-shaped inclusion be considered with the revolution axis taken as the third axis of coordinates and with semi-diameters $d_{1}=d_{2}=d, d_{3}$ $<<$. The inclusion has an isotropic permeability $k^{*}$ embedded in the matrix of isotropic permeability $k$. The fluid velocity $\underline{v}$ in the inclusion is found to be uniform and its value can be deduced, for instance, from the results given by Shafiro and Kachanov [[11]. We consider the limit when $\varepsilon=d_{3} / d \rightarrow 0$ and $k^{*} \rightarrow \infty$. The contribution of the inclusion to the equivalent permeability of the material is made through the integral of the fluid velocity in the inclusion:

$$
\underline{Q}=\int_{\Omega^{*}} \underline{v}(\underline{\xi}) d \xi
$$

This integral is a linear function of the far-field gradient $\underline{A}$ :

$$
Q=-\Psi \cdot \underline{A}
$$

Shafiro \& Kachanov [[11] gave the following general expression of $\Psi$ for an arbitrary value $k^{*}$ of the inclusion and $\varepsilon$ :

$$
\Psi=\frac{\Omega^{*}}{\Omega}\left(k^{*}-k\right)\left\{\left[1+\frac{k^{*}-k}{k} \frac{\pi \varepsilon}{4}\right]^{-1}(\boldsymbol{\delta}-\underline{n} \otimes \underline{n})+\left[1+\frac{k^{*}-k}{k}\left(1-\frac{\pi \varepsilon}{2}\right)\right]^{-1} \underline{n} \otimes \underline{n}\right\}
$$

where, $\Omega$ and $\Omega^{*}$ represent respectively the matrix reference volume and the inclusion volume.

Considering the limiting case of a superconducting crack by taking the limit $\varepsilon \rightarrow 0$ and $k^{*} \rightarrow \infty$ Shafiro \& Kachanov [[11] proposed the following expression for this limit:

$$
\Psi=\frac{16}{3} \vee R^{3} k(\boldsymbol{\delta}-\underline{n} \otimes \underline{n})
$$

where, $v$ is the crack density. Nevertheless, a careful study shows that the limit is not unique and depends on the value of $\varepsilon k^{*}$ which is degenerate when $\varepsilon \rightarrow 0$ and $k^{*} \rightarrow \infty$. Let us suppose that $k^{*}=b k / \varepsilon$ where $b$ is a fixed ratio, hence, $\varepsilon k^{*}=b k$ remains constant when $\varepsilon \rightarrow 0$ and $k^{*} \rightarrow \infty$. The limit of $\Psi$ from the Shafiro \& Kachanov [[11], is obtained to be:

$$
\Psi=\frac{16}{3} \vee R^{3} k \frac{b}{b+(4 / \pi)}(\mathbf{I}-\underline{n} \otimes \underline{n})
$$

The limit expression (47) corresponds then to the assumption $b \rightarrow \infty$. 


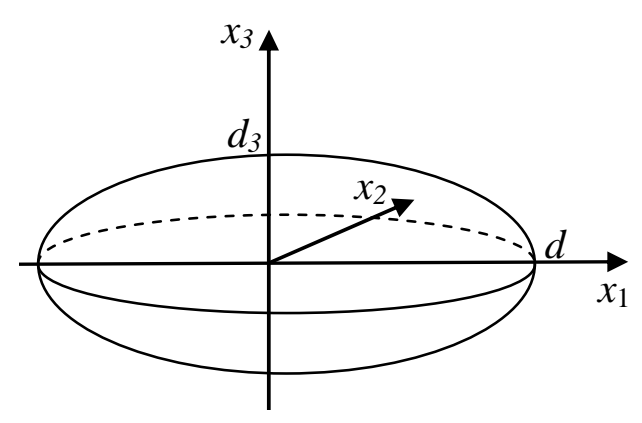

Figure 6: Darcy ellipsoidal inclusion

Let us consider now the ellipsoidal inclusion having $k^{*}$ tends to infinity and $d_{3}$ tends to zero. At the boundary, we note $v^{\mathrm{m}}$ the velocity in the matrix and $\underline{v}^{\mathrm{i}}$ the velocity in the inclusion. The velocity in the inclusion is constant for ellipsoidal inclusion. We note $\underline{v}=v \underline{e}_{1}$. At the boundary, the mass balance yields $\underline{v}^{\mathrm{i}} \cdot \underline{n}=\underline{v}^{\mathrm{m}} \cdot \underline{n}$, where $\underline{n}$ is the unit normal vector to the ellipsoid, and then, $\underline{v}^{\mathrm{m}} \cdot \underline{n}=v n_{1}$ where $n_{1}=\frac{\varepsilon x_{1}}{\sqrt{d^{2}-r^{2}\left(1-\varepsilon^{2}\right)}}$.

The general value of velocity $v$ in the inclusion, for small ratio $\varepsilon=d_{3} / d$, can be deduced from the expression given by Shafiro \& Kachanov [[11] for the integral (44) since $v$ is constant in the inclusion. We find, in first order development with respect to $\varepsilon$ :

$$
v=-\frac{k^{*}-k}{1+\frac{\pi \varepsilon}{4} \frac{k^{*}-k}{k}}
$$

The equivalent infiltration vector in the crack section is deduced from the relation (3) where the thickness $e$ is given, for the ellipsoidal inclusion, by $e=\varepsilon \sqrt{d^{2}-r^{2}}$ and $v$ is constant and given by (49). The limit of the equivalent infiltration $q=2 e v$ for $\varepsilon \rightarrow 0$ is found to be:

$$
\lim _{\varepsilon \rightarrow 0} q=-\frac{8 k c}{\pi c+8 d k} \sqrt{d^{2}-r^{2}}
$$

where, $c=2 d_{3} k^{*}$. We note that for the limit case $c \rightarrow \infty$, the equation (50) yields a solution different from that given by (37). As a matter of fact, it yields only the expression of $q^{0}$ in the equation (19) for circular disc $\left(\phi_{1}=\pi / 4 d\right)$, which is not the complete solution for infiltration in the Poiseiulle's crack since it misses the complementary part $\underline{\omega}$.

It can also be checked that the expression (50) does not satisfy the equation (11). It is then deduced that, in the general case of finite conductivity $c$, the solution of flow in the crack with Poiseuille's law is not given as the limit of thickness vanishing ellipsoidal inclusions with Darcy's law. This implies also that the pressure fields in the inclusion and the matrix are different for the two problems.

Only in the case of superconducting crack, the pressure fields in the two problems are the same since the pressure is constant in the crack or the inclusion. In this case, the integral of $q$ over the inclusion (determining the contribution to the effective permeability, see the next section) is also the same for the two models since it depends only on $q^{0}$ and not on the complementary part $\underline{\omega}$. 
In conclusion, the limit of the flattened inclusion with Darcy's flow does not give in the general case the same result of pressure and infiltration fields that the model of Poiseuilletype crack.

\section{Application to the effective permeability of cracked porous materials}

The effective permeability $\hat{\boldsymbol{k}}$ of the heterogeneous domain is calculated from the average velocity and average pressure gradient in this domain. For a domain $\Omega$ containing a distribution of zero-thickness fractures $\Gamma^{j}$, the equivalent average velocity is defined by:

$$
\underline{V} \equiv \frac{1}{\Omega}\left[\int_{\Omega} \underline{v} d \omega+\sum_{j} \int_{\Gamma^{j}} \underline{q} d s\right]
$$

It can be shown that if a pressure condition corresponding to a uniform macroscopic gradient is applied on the boundary of $\Omega$, i.e., $p(\underline{x})=\underline{A} \cdot \underline{x}$ on $\partial \Omega$, the average pressure gradient $\underline{G}$ is equal to $\underline{A}$ :

$$
\underline{G}=\frac{1}{\Omega} \int_{\Omega} \underline{\nabla} p d \Omega=\underline{A}
$$

We assume that the permeability tensor of the porous matrix is constant and equal to $\boldsymbol{k}$. Introducing (1) and (52) into (51) and using $\underline{V}=-\hat{\boldsymbol{k}} \cdot \underline{G}$ yield:

$$
\hat{\boldsymbol{k}}=\boldsymbol{k}+\boldsymbol{k}^{\mathrm{f}}
$$

where, $\boldsymbol{k}^{\mathrm{f}}$ is the contribution of the cracks to the effective permeability obtained by the following relationship:

$$
\frac{1}{\Omega} \sum_{j} \int_{\Gamma^{j}} \underline{q} d s=-\boldsymbol{k}^{\mathrm{f}} \cdot \underline{A}
$$

The dilute Mori-Tanaka scheme estimates the left-hand side of (54) as the sum of the integral of $q$ over each individual crack by neglecting the effects of cracks interactions. As seen in section 3 , the infiltration in the crack is expressed by $q=q^{0}+\underline{\omega}$. According to (25), $\underline{\omega}$ can be written as: $\underline{\omega}=\underline{e}_{3} \times \nabla \varphi$. Using this relation and (28), we calculate:

$$
\int_{D} \underline{\omega}(\underline{\xi}) d s=-\underline{e}_{3} \wedge \int_{D} \underline{\nabla} \varphi d s=-\underline{e}_{3} \wedge \int_{\partial D} \varphi \cdot \underline{n} d l=-\varphi_{0} \int_{\partial D} \underline{t} d l=0
$$

Therefore, only $q^{0}$ contributes to the integral over the crack surface. From the results given in Section 3, we deduce:

$$
\underline{Q}=\int_{\Gamma} \underline{q}(\underline{\xi}) d s=\int_{\Gamma} \underline{q}^{0}(\underline{\xi}) d s=-\frac{4 k}{3} S_{\Gamma} \boldsymbol{\Phi} \cdot \underline{A}
$$


where, the tensor $\boldsymbol{\Phi}$ defined by (40) and $S_{\Gamma}=\pi d_{1} d_{2}=\pi /|\boldsymbol{B}|^{1 / 2}$ represents the area of the ellipse $\Gamma$. Comparing this relation to (54), the Mori-Tanaka estimate of $\boldsymbol{k}^{\mathrm{f}}$ for an isotropic matrix containing a family of elliptical cracks is found as follows:

$$
\boldsymbol{k}^{\mathrm{f}}=\frac{4}{3} \boldsymbol{v} k<S_{\Gamma} \boldsymbol{\Phi}>
$$

where, $v$ is the crack density, i.e: number of cracks (centres) per unit volume and $\left\langle S_{\Gamma} \boldsymbol{\Phi}\right\rangle$ is the average value of $S_{\Gamma} \Phi$ calculated over the cracks population. For an isotropic distribution of circular cracks with radius $R$, we find:

$$
\boldsymbol{k}^{\mathrm{f}}=\frac{32}{9} k v R^{3} \boldsymbol{\delta}
$$

and the following expression for the dilute Mori-Tanaka effective permeability:

$$
\hat{k}^{M T}=k\left(1+\frac{32}{9} \vee R^{3}\right)
$$

The self-consistent estimate is obtained by replacing, when calculating the contribution of cracks to the effective permeability in equation (58), the matrix permeability by the unknown effective permeability. Therefore, equation (59) leads to the following equation for the selfconsistent estimation $\hat{k}^{s c}$ :

$$
\hat{k}^{s c}=k+\frac{32}{9} \hat{k}^{s c} v R^{3}
$$

Furthermore,

$$
\hat{k}^{s c}=\frac{k}{1-\frac{32}{9} v R^{3}}
$$

The dilute Mori-Tanaka (59) estimation is found to give the first order development for small $v$ of the self-consistent estimation (61) (Figure 7). By introducing the dimensionless crack density $\bar{v}=\frac{4}{3} \pi \nu R^{3}$, the self-consistent estimation becomes singular for a critical value $\bar{v}=\frac{3 \pi}{8} \approx 1.18$. The model is valid only for small values of $v$ and we do not think a physical signification can be attached to this singularity that appears for relatively high values of crack density.

It is worth noting that occasionally this singularity has been related to the percolation limit of the cracks network [[5]. However, Dormieux and Kondo [[5] obtained a different critical value for self-consistent model, $\bar{v}=\frac{3 \pi}{4} \approx 2.36$. The percolation threshold has sometimes been studied numerically. When the domain size is finite, some fracture network configuration percolate and some do not for a given density. It can be only defined a percolation threshold $\bar{v}_{c}$ for the limit of infinite size of domain. Studying the hexagonal fracture networks with isotropic distribution, Bogdanov et al. [[3] found that there is rarely 
percolation for finite fracture network with $\bar{v} \leq 0.42$; percolating and nonpercolating fracture network coexist for the range of densities $0.42 \leq \bar{v} \leq 1.70$ and almost all the fracture are connected when $\bar{v} \geq 1.70$. Huseby et al. [[22] gave a more accurate result for the percolation threshold, $\bar{v} \geq 1.00$ for infinite matrix, which is very close to the critical value obtained in this work by the self-consistent scheme.

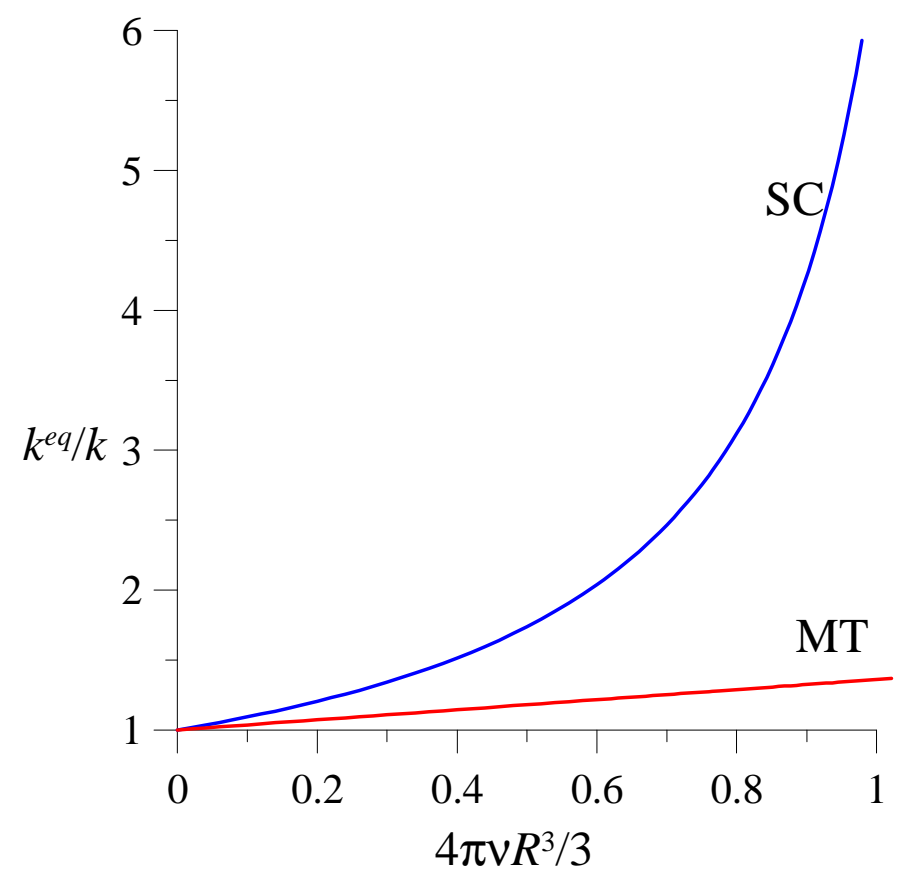

Figure 7: Dilute Mori-Tanaka and self-consistent estimates of effective permeability

\section{Extension to anisotropic matrix}

The linear transformation presented in Pouya [[12] can be used to extend the solutions obtained in the previous sections for elliptical crack in an isotropic matrix to the case of anisotropic matrices. Considering a reversible tensor $\boldsymbol{M}$, we define the transformed variables $\underline{\tilde{x}}, \underline{\tilde{v}}(\underline{\tilde{x}})$ and $\tilde{p}(\underline{\tilde{x}})$ as follows:

$$
\underline{\tilde{x}}=\boldsymbol{M} \cdot \underline{x}, \quad \underline{\tilde{v}}(\underline{\tilde{x}})=\boldsymbol{M} \cdot \underline{v}(\underline{x}), \quad \tilde{p}(\underline{\tilde{x}})=p(\underline{x})
$$

Therefore, $\tilde{x}=\boldsymbol{M} . \underline{x}$ transforms the domain $\Omega$ to a domain $\tilde{\Omega}$, the cracks $\Gamma$ to a new geometry $\tilde{\Gamma}$ The flow problem is then transformed to a new problem corresponding to matrix permeability.

Let us consider an infinite homogeneous porous medium embedding an elliptical crack with a geometry defined by two principal axes $\underline{e}_{1}$ and $\underline{e}_{2}$ and half-diameters $d_{1}$ and $d_{2}$. A pressure gradient $\underline{A}$ is imposed at the infinite boundary of the domain. We refer to Pouya [[12] for the complete set of transformation relations for different variables in which the tensor of permeability is taken the following expression:

$$
\tilde{\boldsymbol{k}}=\boldsymbol{M} \boldsymbol{k} \boldsymbol{M}^{T}
$$


The transformation to the problem studied in previous section is applied by taking $\boldsymbol{M}=\sqrt{\boldsymbol{k}^{-1}}$. In the transformed problem, we find an isotropic matrix with unit permeability: $\tilde{\boldsymbol{k}}$ $=\boldsymbol{\delta}$. The equation of the elliptical crack $\xi \cdot \mathbf{B} \cdot \underline{\xi}=1$ is equivalent to $\underline{\xi} \cdot \boldsymbol{M}^{-1, \mathrm{~T}} \boldsymbol{B} \boldsymbol{M}^{-1} \cdot \underline{\xi}=1$ or $\underline{\tilde{\xi}} . \tilde{\boldsymbol{B}} . \underline{\xi}=1$ with:

$$
\tilde{B}=\sqrt{k} B \sqrt{k}
$$

The tensor $\widetilde{\boldsymbol{B}}$ defines the geometry of the crack in the transformed problem. In order to calculate the half-diameters $\tilde{d}_{1}, \tilde{d}_{2}$ and the principal directions $\underline{\underline{e}}_{1}, \underline{\tilde{e}}_{2}$ (unit vectors) of the transformed ellipse, we need to write:

$$
\tilde{\mathbf{B}}=\tilde{\lambda}_{1} \underline{\tilde{e}}_{1} \otimes \underline{\tilde{e}}_{1}+\tilde{\lambda}_{2} \underline{\tilde{e}}_{2} \otimes \underline{\tilde{e}}_{2}
$$

The expressions $\tilde{\lambda}_{1}$ and $\tilde{\lambda}_{2}$, and of $\tilde{\phi}_{1}$ and $\tilde{\phi}_{1}$ that can be deduced from $\tilde{\lambda}_{1}$ and $\tilde{\lambda}_{2}$ by the same relations (16) are required for writing the solutions of pressure and velocity in the transformed problem. However, if only the pressure field in the matrix in equation (41) or the application to effective permeability in equation (56) is envisaged, it is easier to express directly $\boldsymbol{\Phi}$ as function of $\boldsymbol{B}$ (see Appendix C):

$$
\boldsymbol{\Phi}=f \boldsymbol{\delta}+g \boldsymbol{B}
$$

where, $f$ and $g$ are scalar functions of $B=\boldsymbol{B}: \boldsymbol{\delta}$ and $\beta=|\boldsymbol{B}|$.

As a matter of fact, the pressure transformation $\tilde{p}(\underline{\tilde{x}})=p(\underline{x})$ leads to $\underline{\tilde{A}} \cdot \underline{\tilde{x}}=\underline{A} \cdot x$ at infinity; hence, $\underline{\tilde{A}}=\sqrt{\mathbf{k}} \cdot \underline{A}$. Moreover, $\underline{\tilde{q}}^{0}(\underline{\tilde{\xi}})$ in the transformed problem is given by (39) such as:

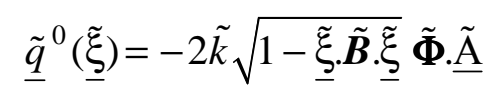

where $\tilde{k}=1$ and:

$$
\tilde{\boldsymbol{\Phi}}=f(\tilde{B}, \tilde{\boldsymbol{\beta}}) \boldsymbol{\delta}+g(\tilde{B}, \tilde{\boldsymbol{\beta}}) \tilde{\boldsymbol{B}}
$$

The general transformation rule for infiltration vectors is (see in Pouya [[12]):

$$
\underline{\tilde{q}}^{0}=\frac{1}{\sqrt{\underline{n} \cdot \mathbf{k} \cdot \underline{n}}} \sqrt{\mathbf{k}^{-1}} \cdot \underline{q}^{0}
$$

Replacing by these values function of initial variables yields:

$$
\underline{q}^{0}(\underline{\xi})=-2 \sqrt{\underline{n} \cdot \mathrm{k} \cdot \underline{n}} \sqrt{1-\underline{\xi} \cdot \boldsymbol{B} \cdot \underline{\xi}} \sqrt{\mathbf{k}} \tilde{\boldsymbol{\Phi}} \cdot \sqrt{\mathbf{k}} \underline{A}
$$

with $\tilde{\boldsymbol{B}}$ given by (64), $\tilde{B}=\tilde{\boldsymbol{B}}: \boldsymbol{\delta}=\boldsymbol{k}: \boldsymbol{B}$ and $\tilde{\beta}=|\tilde{\boldsymbol{B}}|=|\boldsymbol{k}||\boldsymbol{B}|$ and the expressions the functions $f$ and $g$ given in Appendix C. In this way, $q^{0}(\xi)$ is calculated explicitly for arbitrary elliptical 
shaped crack in a matrix with arbitrary anisotropic permeability $\boldsymbol{k}$. The pressure field in the matrix is deduced from:

$$
p(\underline{x})=\underline{A} \cdot \underline{x}+\frac{1}{4 \pi \sqrt{|\boldsymbol{k}|}} \int_{D} \frac{q^{0}(\underline{\xi}) \cdot \boldsymbol{k}^{-1}(\underline{\xi}-\underline{x})}{\left\|(\underline{\xi}-\underline{x}) \cdot \boldsymbol{k}^{-1} \cdot(\underline{\xi}-\underline{x})\right\|^{3 / 2}} d \xi
$$

For extension of the results concerning the effective permeability, it is sufficient to write (57) for the transformed problem:

$$
\tilde{\boldsymbol{k}}^{\mathrm{f}}=\frac{4}{3} \tilde{\mathrm{v}} \tilde{k}<\tilde{S}_{\Gamma} \tilde{\boldsymbol{\Phi}}>
$$

Noting that $\tilde{k}=1, \quad \tilde{v}=v /|\boldsymbol{M}|=v \sqrt{|\boldsymbol{k}|}, \quad \tilde{S}_{\Gamma}=\pi / \sqrt{|\tilde{\boldsymbol{B}}|}, \quad \boldsymbol{k}^{\mathrm{f}}=\sqrt{\boldsymbol{k}} \tilde{\boldsymbol{k}}^{\mathrm{f}} \sqrt{\boldsymbol{k}}$, and using the expressions obtained here above for $\tilde{\boldsymbol{\Phi}}$ and $\tilde{\boldsymbol{B}}$, we find:

$$
\boldsymbol{k}^{\mathrm{f}}=\frac{4}{3} \mathrm{v} \sqrt{\boldsymbol{k}}<S_{\Gamma} \tilde{\Phi}>\sqrt{\boldsymbol{k}}
$$

Substituting (72) into (53) allows us to determine the effective permeability of a porous body with anisotropic matrix permeability $\boldsymbol{k}$ containing a distribution of elliptical superconducting cracks by using the Mori-Tanaka or the self-consistent estimate.

In practice, field observation permits us to determine the cracks geometries (size, aperture and orientation) and also their density [[23]. An estimation of the crack conductivity is also required that can be obtained by a Poiseuille's model. However, in our work, only superconducting disc-shaped cracks are considered. The crack network is assumed to be randomly distributed. With these data, the models given in Section 6 are suitable to estimating the effective permeability.

\section{Conclusions and perspectives}

The equation governing the steady state flow around a crack surface in an infinite porous body, deduced from previous investigations [[12], was considered in this paper. An analytical solution was derived for this equation in the case of a superconducting and elliptical disc shaped crack.

Cracks have been very often modelled as flattened ellipsoidal inclusions obeying Darcy's law because for this problem analytical results are available. The question raises then if this model can represent well a crack with zero thickness and obeying to the Poiseuille's law. In the literature, it is often implicitly responded positively to this question: it is assumed that the flow field in and around a crack with Poiseille's law can be obtained as the limiting case of the flow field in and around a flattened ellipsoidal inclusion with Darcy's law when its thickness tends to zero. The results obtained in the present work allowed the comparison. We could show that the equivalence between the two models does not hold in the general case. Only some partial results in restricted cases, mainly the case of superconducting cracks, can be obtained as a limit case. In general, for cracks with finite conductivity, the pressure gradient through the mean crack surface is constant in ellipsoidal inclusion model but not in 
Poiseuille's type crack. These results change fundamentally the vision we can have for the flow in a Poiseuille's type crack.

The results obtained for a single crack have been used to estimate the effective permeability of cracked porous materials. The dilute Mori-Tanaka model is a very simple model that does not take into account the interaction between cracks. The expression of the effective permeability is a linear function of the crack density according to this model, for anisotropic matrix permeability and elliptical disc cracks. The crack interaction is taken into account implicitly in the self consistent model. We established the exact expression of the effective permeability according to this model for circular cracks in a matrix with isotropic permeability. This non linear model presents a singularity for a critical value of crack density that is sometimes interpreted as representing the percolation threshold of crack network. This critical value is then compared to analogous values given in the literature, and found to be very close to that obtained by numerical simulation for the percolation threshold of crack networks.

The analytical solutions given in this paper open the way for deeper theoretical investigations. The effective permeability of micro-cracked materials can take benefit from these results as well as the study of crack interactions within a porous body. Also it is worth reminding that a fundamental assumption underlying the present work was the infinite transversal conductivity of cracks that removes the possibility of pressure jump between to sides of the crack. But in some physical cases, cracks act as an impervious membrane with a pressure discontinuity across the crack surface [[3, [10, [24]. The approach used by Martin [[24] could be used in this case to extend some of the results of the present paper to these types of cracks.

Analytical infiltration solution through a single crack obtained here was restricted to the case of superconducting cracks. For the case of Poiseiulle-type cracks with finite conductivity, semi-analytical and numerical calculations are required that we have investigated in an ongoing work and we hope present the results in a future paper. Moreover, numerical study allows us to take into consideration explicitly the crack interaction and the crack intersection. The three-dimensional effective permeability model that can be obtained in this way would allow extending the two-dimensional application to $\mathrm{CO} 2$ storage [[25] to a three-dimensional modelling.

\section{Appendix A: Integral calculation for a point inside the elliptical disc}

Replacing by the (14) in the main text in (12), the following equation (A.1) is found in which $\boldsymbol{B}$ is diagonal with eigenvalues $\lambda_{1}, \lambda_{2}$, and $\xi . \boldsymbol{B} \cdot \xi=\lambda_{1} \xi_{1}{ }^{2}+\lambda_{2} \xi_{2}{ }^{2}$. We use for $\xi$ the polar coordinates $(\rho, \theta)$ in the local coordinate system having $\underline{x}$ as origin (see Figure 3 ). We have $\xi_{1}=x_{1}+\rho \cos \theta, \xi_{2}=x_{2}+\rho \sin \theta$. Thus, we have:

$$
I_{1}=\int_{D} \frac{\sqrt{1-\underline{\xi} \cdot \boldsymbol{B} \cdot \underline{\xi}}}{\|\underline{\xi}-\underline{x}\|^{3}}\left(\xi_{1}-x_{1}\right) d \xi=\int_{\theta=0}^{2 \pi} \int_{\rho=0}^{\rho_{m}} \frac{\sqrt{1-\underline{\xi} \cdot \boldsymbol{B} \cdot \underline{\xi}}}{\rho} d \rho \cos \theta d \theta
$$

It is obvious that this integral must be considered as the Cauchy principal value: 
$I_{1}=\lim _{\varepsilon \rightarrow 0} I_{1 \varepsilon} \quad, \quad I_{1 \varepsilon}=\int_{\theta=0}^{2 \pi} J_{\varepsilon}(\theta) \cos \theta d \theta$

where:

$$
J_{\varepsilon}(\theta)=\int_{\varepsilon}^{\rho_{m}} \frac{\sqrt{1-\underline{\xi} \cdot \boldsymbol{B} \cdot \underline{\xi}}}{\rho} d \rho=\frac{\gamma}{e} \int_{\varepsilon}^{\rho_{m}} \sqrt{1-(e \rho+g)^{2}} \frac{d \rho}{\rho}
$$

with:

$$
\begin{aligned}
& \gamma=\sqrt{\lambda_{1} \cos ^{2} \theta+\lambda_{2} \sin ^{2} \theta}, \quad H=\sqrt{\gamma^{2}-\lambda_{1} \lambda_{2}\left(x_{1} \sin \theta+x_{2} \cos \theta\right)^{2}} \\
& e=\frac{\gamma^{2}}{H}, \quad g=\frac{\lambda_{1} x_{1} \cos \theta+\lambda_{2} x_{2} \sin \theta}{H}
\end{aligned}
$$

We take the variable $\alpha$ :

$$
\sin \alpha=e \rho+g
$$

When $\rho$ varies between $\varepsilon$ and $\rho_{\mathrm{m}}, \alpha$ varies between $\alpha_{\varepsilon}=\operatorname{Arcsin}(\rho \varepsilon+g)$ to $\pi / 2$. In this interval, $\cos \alpha \geq 0$, and variable change $\rho \rightarrow \alpha$ allows calculating:

$$
J_{\varepsilon}(\theta)=\frac{\gamma}{e} \int_{\alpha=\alpha_{\varepsilon}}^{\pi / 2}\left(\frac{1-g^{2}}{\sin \alpha-g}-(\sin \alpha+g)\right) d \alpha
$$

and find:

$$
J_{\varepsilon}(\theta)=\frac{\gamma\left(1-g^{2}\right)}{e} F_{\varepsilon}(\theta)-\frac{\gamma}{e}\left[\cos \alpha_{\varepsilon}+g\left(\pi / 2-\alpha_{\varepsilon}\right)\right]
$$

Where:

$$
F_{\varepsilon}(\theta)=\int_{\alpha=\alpha_{\varepsilon}}^{\pi / 2} \frac{1}{\sin \alpha-g} d \alpha
$$

Now we can write:

$$
I_{1, \varepsilon}=\int_{\theta=0}^{\pi}\left[J_{\varepsilon}(\theta)-J_{\varepsilon}(\theta+\pi)\right] \cos \theta d \theta
$$

When changing $\theta$ to $\theta+\pi$, the functions $\gamma, e, H$ remain unchanged, but $g$ is changed to $-g, \alpha_{\varepsilon}$ to $\alpha_{\varepsilon}^{\prime}=\operatorname{Arcsin}(\rho \varepsilon-g)$ and the $u_{\varepsilon}$ to $u_{\varepsilon}^{\prime}=\tan \left(\alpha_{\varepsilon}^{\prime} / 2\right)$. It can be shown that:

$$
\lim _{\varepsilon \rightarrow 0}\left[F_{\varepsilon}(\theta)-F_{\varepsilon}(\theta+\pi)\right]=\int_{-\pi / 2}^{\pi / 2} \frac{1}{\sin \alpha-g} d \alpha
$$


where the integral in the right-hand side must be considered as the Cauchy principal value and can be shown to take the following values:

$$
\left\{\begin{aligned}
\int_{-\pi / 2}^{\pi / 2} \frac{1}{\sin \alpha-g} d \alpha & =\frac{-\pi}{g \sqrt{1-\frac{1}{g^{2}}}} & \text { if }|g|>1 \\
& =0 & \text { if }|g|<1
\end{aligned}\right.
$$

By using this result (with $|g|<1$ ) and the limits $\lim _{\varepsilon \rightarrow 0} \alpha_{\varepsilon}=-\lim _{\varepsilon \rightarrow 0} \alpha_{\varepsilon}^{\prime}=\operatorname{Arcsin}(g)$, we find:

$$
I_{1}=-\pi \int_{\theta=0}^{\pi} \frac{\gamma g}{e} \cos \theta d \theta
$$

Replacing by (A.4) for $\gamma, g$ and $e$ in this expression and after integration we obtain:

$$
I_{1}=-2 \pi \lambda_{1} x_{1} \int_{\theta=0}^{\pi / 2} \frac{\cos ^{2} \theta}{\sqrt{\lambda_{1} \cos ^{2} \theta+\lambda_{2} \sin ^{2} \theta}} d \theta=-2 \pi \phi_{1}\left(\lambda_{1}, \lambda_{2}\right) x_{1}
$$

where the function $\phi_{1}\left(\lambda_{1}, \lambda_{2}\right)$ is defined by the equation (16) in the main text.

Furthermore, using the same notation with $x_{1}=x_{2}=0$, and thus, $g=0$ and $e=\gamma$, we have:

$$
\int_{\theta=0}^{2 \pi} \int_{\rho=0}^{\rho_{m}} \sqrt{1-\underline{\xi} . \boldsymbol{M}} \underline{\underline{\xi}} \rho d \rho d \theta=\int_{\theta=0}^{2 \pi} \int_{\rho=0}^{1 / \gamma} \sqrt{1-\gamma^{2} \rho^{2}} \rho d \rho d \theta=\int_{\theta=0}^{2 \pi} \frac{d \theta}{3 \gamma^{2}}=\frac{2 \pi}{3 \sqrt{\lambda_{1} \lambda_{2}}}
$$

\section{Appendix B: Integral calculation for a point outside the circular disc}

As seen in Figure 4 in the text, the integral in the left-hand side of (42) can be re-written in the polar coordinate system as follows:

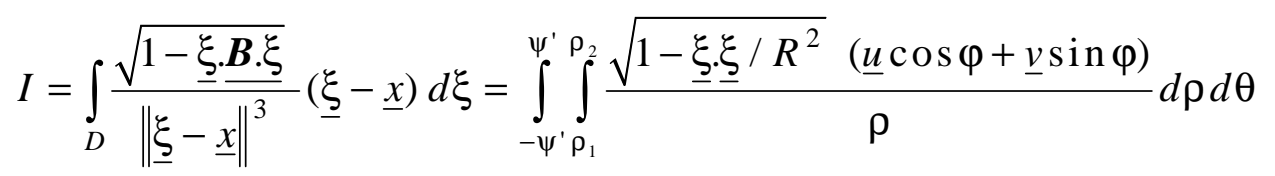

where:

$$
\psi^{\prime}=\psi / 2
$$

Also, we can write $\sqrt{1-\underline{\xi} . \underline{\xi} / R^{2}}=\frac{1}{e R} \sqrt{1-(e \rho+g)^{2}} \quad$ with: $e=\frac{1}{\sqrt{R^{2}-\|\underline{x}\|^{2} \sin ^{2} \varphi}}$ and $g=-e\|\underline{x}\| \cos \varphi$. Then the symmetries with respect to $\varphi \rightarrow-\varphi$ imply that we can write: $I=\left(\int_{-\psi^{\prime}}^{\psi^{\prime}} \frac{1}{e R} J(\varphi) \cos \varphi d \varphi\right) \underline{u} \quad$ where: $J(\varphi)=\int_{\rho_{1}}^{\rho_{2}} \frac{\sqrt{1-(e \rho+g)^{2}}}{\rho} d \rho$ 
For the same variable change (A.5), $\alpha$ varies between $-\pi / 2$ to $\pi / 2$ when $\rho$ varies between $\rho_{1}$ and $\rho_{2}$. By the same method than in the previous case, and using (A.11) with, this time, $|g|>1$, we calculate:

$\frac{1}{e R} J(\varphi) \cos \varphi=\left(\sqrt{1-\frac{1}{g^{2}}}-1\right) \frac{g}{e} \pi=-\frac{\pi}{\sin \psi^{\prime}}\left(\cos \psi^{\prime}-\cos \varphi\right) \cos \varphi$

where, $\sin \psi^{\prime}=\frac{R}{\|\underline{x}\|}$. Thus, by integration and using $\underline{u}=\frac{\sin \psi^{\prime}}{R} \underline{x}$, we find finally,

$I=\left(\int_{-\psi^{\prime}}^{\psi^{\prime}} \frac{1}{e R} J(\varphi) \cos \varphi d \varphi\right) \underline{u}=\frac{\pi}{2 R}\left(2 \psi^{\prime}-\sin 2 \psi^{\prime}\right) \underline{x}=\frac{\pi}{2 R}(\psi-\sin \psi) \underline{x}$

\section{Appendix C: Linear relation between two tensors $B$ and $\Phi$}

For a general second order tensor $\boldsymbol{B}$, we note $B=\boldsymbol{B}: \boldsymbol{\delta}$ and $\beta=|\boldsymbol{B}|$ (determinant of $\boldsymbol{B}$ ). For $\boldsymbol{B}=\lambda_{1} \underline{e}_{1} \otimes \underline{e}_{1}+\lambda_{2} \underline{e}_{2} \otimes \underline{e}_{2}$ we have $B=\lambda_{1}+\lambda_{2}$ and $\beta=\lambda_{1} \lambda_{2}$. Therefore, $\lambda_{1}$ and $\lambda_{2}$ are the solutions of:

$$
\lambda_{1,2}=\frac{B \mp \sqrt{\boldsymbol{B}: \boldsymbol{B}-4 \beta}}{2}
$$

For a given $\boldsymbol{B}$, we define: $\boldsymbol{\Phi}=\phi_{1}^{-1} \underline{e}_{1} \otimes \underline{e}_{1}+\phi_{2}^{-1} \underline{e}_{2} \otimes \underline{e}_{2}$ where $\phi_{1}$ and $\phi_{2}$ verify equation (17) in the main text. i.e:

$$
\phi_{1}+\phi_{2}=\frac{\sqrt{\lambda_{2}-\lambda_{1}}}{\lambda} E(\lambda), \quad \frac{1}{1-\lambda^{2}} \phi_{1}+\phi_{2}=\frac{\sqrt{\lambda_{2}-\lambda_{1}}}{\lambda} K(\lambda)
$$

with $\lambda=\sqrt{1-\frac{\lambda_{1}}{\lambda_{2}}}$ and $K(\lambda)$ and $E(\lambda)$ the complete elliptical integrals of first or second kind.

The solutions of $\phi_{1}, \phi_{2}$ can be written as:

$$
\begin{aligned}
& \phi_{1}=\frac{\sqrt{\lambda_{2}-\lambda_{1}}}{\lambda} \cdot \frac{1-\lambda^{2}}{\lambda^{2}}[K(\lambda)-E(\lambda)]=\frac{1}{\chi_{1}(B, \beta)} \\
& \phi_{2}=\frac{\sqrt{\lambda_{2}-\lambda_{1}}}{\lambda}\left[-\frac{1-\lambda^{2}}{\lambda^{2}} K(\lambda)+\frac{1}{\lambda^{2}} E(\lambda)\right]=\frac{1}{\chi_{2}(B, \beta)}
\end{aligned}
$$

Remarking that two tensors $\boldsymbol{\Phi}$ and $\boldsymbol{B}$ have the same principal directions $\underline{e}_{1}, \underline{e}_{2}$, we can write:

$$
\Phi=\sigma \delta+\tau \overline{\mathbf{B}}
$$


where $\sigma, \tau$ are two scalars and $\bar{B}=\boldsymbol{B}-\frac{1}{2} B \boldsymbol{\delta}$. From this relation we deduce that:

$$
\boldsymbol{\Phi}: \boldsymbol{\delta}=3 \sigma, \quad \operatorname{det} \boldsymbol{\Phi}=\sigma^{2}-\frac{\tau^{2}}{4}\left(B^{2}-4 \beta\right)
$$

So, $\phi_{1}$ and $\phi_{2}$ can be determined from the system of equations :

$$
\frac{1}{\phi_{1}}+\frac{1}{\phi_{2}}=3 \sigma, \quad \frac{1}{\phi_{1} \phi_{2}}=\sigma^{2}-\frac{\tau^{2}}{4}\left(B^{2}-4 \beta\right)
$$

From these equations, we determine $\sigma$ and $\tau$ function of $\phi_{1}$ and $\phi_{2}$ and so, finally, function of $B$ and $\beta$ :

$$
\sigma(B, \beta)=\frac{1}{3}\left(\chi_{1}+\chi_{2}\right), \quad \tau(B, \beta)=\frac{\chi_{1}^{2}+\chi_{2}^{2}-7 \chi_{1} \chi_{2}}{9 \alpha^{2}}, \alpha(B, \beta)=\frac{\sqrt{\boldsymbol{B}: \boldsymbol{B}-4 \beta}}{2}
$$

Finally, we define two scalar functions $f$ and $g$ as follows:

$$
f(B, \beta)=\sigma-\frac{1}{2} B \tau, \quad g(B, \beta)=\tau
$$

These allow writing:

$$
\boldsymbol{\Phi}=f \boldsymbol{\delta}+g \boldsymbol{B}
$$

\section{References}

[1] Koudina N., Gonzalez Garcia R., Thovert J.F., Adler P.M., Permeability of three-dimensional fracture networks, Phys. Rev. E, 57 (1998), 4466-4479.

[2] Mourzenko V.V., Thovert J.F., Adler P.M., Macroscopic permeability of three-dimensional fracture networks with power-law size distribution, Phys. Rev. E., 69 (6) (2004), 066307.

[3] Bogdanov I.I., Mourzenko V.V., Thovert J.F., Adler P.M., Effective permeability of fractured porous media in steady state flow, Water Resources Research, 39(1) (2003), 1023, doi:10.1029/2001WR000756.

[4] Arbogast T., Douglas J., Hornung U., Derivation of the double-porosity model of single phase flow via homogeneisation theory, SIAM, J. Math. Anal., 21 (1990), 823-836.

[5] Dormieux L., Kondo D., Approche micromécanique du couplage perméabilité-endommagement, C.R. Mecanique 332 (2004), 135-140.

[6] Pouya A., Fouché O., Permeability of 3D discontinuity networks: New tensors from boundary-conditioned homogenisation, Advances in Water Resources, 32 (2009), 303-314.

[7] Zimmerman R., Effective conductivity of a two-dimensional medium containing elliptical inhomogeneities. Proc. Roy. Soc. Ser. A 452(1950) (1996), 1713-1727. 
[8] Markov M., Kazatchenko E., Mousatov A., Pervago E., Permeability of the Fluid-Filled Inclusions in Porous Media, Transport in porous media, 84(2) (2010), 307-317.

[9] Simunek J., Jarvis N.J., van Genuchten M.Th., Gärdenäs A., Review and comparison of models for describing non-equilibrium and preferential flow and transport in the vadose zone. Journal of Hydrology 272 (2003) 14-35.

[10] Manzocchi T., Childs C., Walsh J.J., Faults and fault properties in hydrocarbon flow models, Geofluids 10 (2010), 94-113.

[11] Shafiro B., Kachanov M., Anisotropic effective conductivity of material with nonrandomly oriented inclusions of divers ellipsoidal shapes. Journal of applied physics 87(12) (2000), 8561-8569.

[12] Pouya, A., Three-dimensional flow in fractured porous media: a potential solution based on singular integral equations. Advances in Water Resources 35 (2012) 30-40.

[13] Liolios P.A., Exadaktylos G.E.,, A solution of steady-state fluid flow in multiply fractured isotropic porous media, International Journal of Solids and Structures 43 (2006), 3960-3982.

[14] Pouya, A., Ghabezloo S., Flow around a crack in a porous matrix and related problems. Transport in Porous Media 84(2) (2010), 511-532.

[15] Green, A.E., Sneddon, I.N., The distribution of stress in the neighborhood of a flat elliptic crack in an elastic solid. Proc. Cambridge Phil. Soc. 46 (1950), 159-164.

[16] Selvadurai, A.P.S., On the hydraulic intake shape factor for a circular opening located at an impervious boundary: Influence of inclined stratification. International Journal for Numerical and Analytical Methods in Geomechanics 35 (2011), 639-651.

[17] Sneddon I.N., The distribution of stress in the neighborhood of a crack in an elastic solid. - Proceedings of the Royal Society of London, 187A (1946) 229-260.

[18] Selvadurai, A.P.S., Advective transport from a penny-shaped crack and an associated uniqueness theorem. International Journal for Numerical and Analytical Methods in Geomechanics 28 (2004), 191-208.

[19] Guéguen, Y., Palciauskas V., Introduction to Physics of Rocks, Princeton Univ. Press, 1994.

[20] Gentier S., Lamontagne E., Archambault G., Riss J., Anisotropy of flow in a fracture undergoing shear and its relationship to the direction of shearing and injection pressure, Int. J. RockMech. \&Min. Sci. 34:3-4, Paper No. 094 (1997).

[21] Abramowitz M., Stegun I. A., (Eds.). ”The Process of the Arithmetic-Geometric Mean." §17.6 in Handbook of Mathematical Functions with Formulas, Graphs, and Mathematical Tables, 9th printing. New York: Dover, pp. 571 and 598-599, 1972.

[22] Huseby O., Thovert, J.F., Adler P.M., Geometry and topology of fracture systems, J. Phys. A, 30 (1997), 1415-1444.

[23] Gervais F., Modélisation géométrique d'un réseau de fractures dans un massif rocheux stratifié. Application aux carrières marbrières de Comblanchien (Côte d'Or, France), $\mathrm{PhD}$ dissertation, Ecole Nationale Supérieure des Mines de Paris (1993).

[24] Martin P.A., On potential flow past wrinkled discs, Proceedings of the Royal Society A 454 (1998), 22092221.

[25] Seyedi D. M., Ducellier A., Vu M.N. Pouya A., A two-scale model for simulating the hydromechanical behavior of faults during CO2 geological storage operation, $45^{\text {th }}$ US Rock Mechanics/Geomechanics Symposium, San Fransisco, June 26-29, 2011. 